\title{
Ultradian and circadian modulation of dream recall: EEG correlates and age effects
}

\author{
Sarah Laxhmi Chellappa ${ }^{a, b, *}$, Christian Cajochen ${ }^{\text {a }}$ \\ a Centre for Chronobiology, Psychiatric Hospital of the University of Basel, Wilhelm Kleinstrasse 27, Basel, Switzerland \\ b Cyclotron Research Center, University of Liège, Allée du 6 Âout 8, Bat. 30, Sart-Tillman, 4000-Liège, Belgium
}

\section{A R T I C L E I N F O}

\section{Article history:}

Received 7 December 2012

Received in revised form 4 March 2013

Accepted 11 March 2013

Available online $\mathrm{xxxx}$

\section{Keywords:}

Dreaming

NREM sleep

REM sleep

Circadian system

Brain topography

fMRI

\begin{abstract}
A B S T R A C T
Dreaming occurs during non-rapid eye movement (NREM) and rapid eye movement (REM) sleep, which both are regulated by homeostatic, ultradian, and circadian processes. However, the magnitude of how ultradian REM and NREM sleep and its EEG correlates impact onto dream recall remains fairly unknown. In this review, we address three questions: 1 . Is there an ultradian NREM-REM sleep modulation in successful dream recall, which is gated by the circadian clock? 2. What are the key electrophysiological correlates that account for dream recall during NREM and REM sleep and 3. Are there age-related changes in the ultradian and circadian regulation in dream recall and its electrophysiological correlates? Knowledge on the specific frequency and topography NREM and REM sleep differences prior to dream recall may pinpoint to the cerebral correlates that account for this cognitive process, and hint to their possible physiological meaning.
\end{abstract}

(c) 2013 Published by Elsevier B.V.

\section{Introduction}

Dreaming is a universal human experience that offers one of the most unique views to consciousness and cognition, although there is much controversy on what exactly dreaming is. Theoretical definitions encompass simplistic perspectives as "any mental activity occurring in sleep" to specific frameworks as "dreaming represents the conscious awareness of complex brain systems involved in the reprocessing of emotions and memories during sleep" (Stickgold et al., 2001a) to narrower ones, such as "mental activity occurring in sleep characterized by vivid sensorimotor imagery that is experienced as waking reality despite such distinctive cognitive features as impossibility or improbability of time, place, person and actions; emotions, especially fear, elation and anger predominate over sadness, shame and guilt and sometimes reach sufficient strength to cause awakening; memory for even very vivid dreaming is evanescent and tends to fade quickly upon awakening unless special steps are taken to retain it" (Hobson et al., 2000). The functional importance of dreaming still remains a matter of intense debate. Chief among the hypotheses that may explain the relevance of dreaming are the mind-brain reductionism, which suggests that dreaming is a random by-product of underlying REM sleep physiology (Hobson and Mc Carley, 1977). More recently, dreaming has been argued as a

\footnotetext{
Conflict of interest: The authors disclose no conflicts of interest including financial, personal or other relationships with people or organizations that influence this work.

* Corresponding author at: Cyclotron Research Center, University of Liège, Allée du 6 Âout 8, Bat. 30, Sart-Tillman, 4000-Liège, Belgium. Tel.: + 32 43662303; fax: + 32 43662946.

E-mail address: slchellappa@ulg.ac.be (S.L. Chellappa).
}

means for reactivation and consolidation of novel and individuallyrelevant features of prior wake experience (Wamsley et al., 2010; Desseilles et al., 2011). Thus, dreaming may enable the replay of isolated elements (from their original context) for the integration of new features into existing cognitive representations linked to specific brain areas (Schwartz and Maquet, 2002). Another functional reasoning for dreaming to occur is that it may allow the enactment of affective processes by providing an internal activation, which is driven from an individual's affective and emotional history and serving an emotionality-regulation function (Cartwright et al., 1997a, 1997b; Nielsen and Chénier, 1999). Thus, dreaming may stimulate the resolution of emotional conflict and reduce next-day negative mood (Cartwright et al., 1997a).

In this review, we present electroencephalographic correlates of successful dream recall after NREM and REM sleep in healthy young and older volunteers and describe how NREM-REM sleep and circadian rhythmicity modulate dreaming, focusing particularly on our recent data on dream recall, and provide evidence on how it may change in healthy aging. Furthermore, we give an overview of neuroimaging data looking at the cerebral correlates that may account for dreaming, and draw, at the end, perspectives on what dreaming may imply.

\section{Ultradian REM and NREM sleep modulation of dream recall and its EEG correlates}

The neuroscientific basis of dreaming was firstly deemed to be modulated by the ultradian NREM-REM sleep cycle, particularly after the discovery that higher levels of dream recall were strongly connected to prior REM sleep (Aserinsky and Kleitman, 1953). Thus, it was plausible to believe that the specific brain mechanisms of REM 
sleep may hint to the origin of dream processing (Dement and Kleitman, 1957). This assumption leads to the "activation-synthesis" hypothesis, which described the brainstem generators of REM sleep as the basis of dreaming (Hobson and Mc Carley, 1977). This hypothesis triggered numerous studies thereof that aimed at understanding dream generation as a by-product of REM sleep (Cartwright et al., 1997b; Czaya et al., 1973; Foulkes, 1993; Casagrande et al., 1996). Nevertheless, this REM-centric belief has been challenged by compelling evidence of dreaming during NREM sleep (Cavallero et al., 1990; Nielsen, 2000, 2004). Initially, NREM dreaming was ascribed as a memory from a previous REM episode, as argued by the "covert" REM sleep theory, given that the great majority of NREM dream recall was observed during the second part of the night, most likely after a previous REM sleep episode (Nielsen, 2000). This type of assumption stemmed from the fact that NREM dreaming differs from REM dreaming on fundamental aspects, such that dream report lengths are 2 to 5 times longer in REM than NREM sleep episodes (Nielsen, 2004). Besides incidence and length, REM and NREM dreaming are substantially different with respect to its emotional salience. REM sleep dream reports are more emotional, anxious, with obvious visualized different scenes and more socially unacceptable content, while NREM reports are consistently more conceptual and thought-like (Hobson et al., 2000; Stickgold et al., 2001a).

If sleep EEG activity during dreaming reflects the activation or deactivation of particular anatomical pathways related to this process, it may unravel processes related to dreaming (Desseilles et al., 2011). Previous data on EEG spectral power and dream recall have provided mixed results. For instance, EEG beta activity has been shown to increase in NREM sleep stage 2, at the transition from epochs without dream recall to those with distinct recall (Williamson et al., 1986). Furthermore, less EEG power density in the theta range (5$8.5 \mathrm{~Hz}$ ) has been reported for successful recall subsequent to NREM sleep (Cajochen et al., 2003). With respect to REM dreaming, alpha activity (Esposito et al., 2004) and widespread $40-\mathrm{Hz}$ oscillations have been associated to increased dream recall (Llinás and Ribary, 1993), as both may induce large functional states for cognitive processing. Faster oscillations during REM sleep may thus turn out to be an "electrophysiological signature" for dream processing (Llinás and Ribary, 1993). Interestingly, there is also evidence for an inverse relationship between EEG slow-wave activity and dream recall, with higher levels of delta activity associated to no recall conditions during NREM sleep and, particularly, REM sleep (Esposito et al., 2004). Indeed, the inverse association was more robust for REM sleep, indicating that delta power may be a negative index of dream recall. Early studies found similar inverse relationships for more broadly defined frequency bands, such as $4.0-14.0 \mathrm{~Hz}$ (Lehmann et al., 1981). More recently, successful dream recall, after morning awakening from REM sleep, was associated to higher frontal theta $(5-7 \mathrm{~Hz})$ activity, while dream recall following morning awakening from NREM sleep stage 2 resulted in less alpha $(8-12 \mathrm{~Hz})$ activity of the right temporal area (Marzano et al., 2011). These results may possibly hint to a neurophysiological mechanism that underlies encoding and recall of episodic memories, which may remain similar across different sleep states (Marzano et al., 2011). However, the precise EEG spectral correlates of dream recall are still unclear. One likely candidate is the use of different EEG approaches, such as which sleep stages are taken into account (only NREM or REM sleep or both), and which power spectra are computed for the analysis (NREM sleep delta or REM sleep alpha power, and so forth) (Armitage et al., 1989; Esposito et al., 2004). Another possible explanation is the use of different approaches accounting for dreams, such as qualitative (dream content) or quantitative methods (dream recall and number of dreams) (Stickgold et al., 2001b). Another aspect that also remains largely unknown is the role of the circadian timing system on the generation of dreaming. In the next section we address studies that focused on the impact of the circadian timing system on dreaming.

\section{The Tick-tock of dreaming: circadian activation of dream recall}

Circadian-driven changes in cortical activity are a very likely candidate to promote dreaming during NREM sleep and, particularly, during REM sleep (Wamsley et al., 2007). Compelling evidence for circadian influences on dreaming arise from a study in which relationships between circadian factors and dreaming were out of sync (Antrobus et al., 1995). In this study, sleep onset and offset were delayed by $3 \mathrm{~h}$ to create a phase delay of dreaming and to test the hypothesis of circadian influences. REM and NREM dreaming would then presumably occur $3 \mathrm{~h}$ later than usual, thus coinciding with the hypothetical rising phase of core body temperature and with temporally close to the peak of REM sleep propensity. Dream reports were then compared from the phase-delayed condition to control reports from non-delayed sleep (Antrobus et al., 1995). Delayed dream reports were longer and visually more intense, particularly during the latter part of the night as compared to reports collected at the beginning of the night. While REM and NREM sleep differences were observed, both REM and NREM dream reports were affected by the circadian factor independent of sleep state differences. In particular, for visual imagery the circadian effect size ( 0.23 or small) was about $30 \%$ of the ultradian NREM/REM effect size ( 0.70 or large). These findings suggest that the ultradian NREM-REM sleep and circadian sources of cortical and subcortical activation can be independent, but additive, in their effects on dreaming (Antrobus et al., 1995). Further support for the circadian-driven activation of dreaming derives from an ultra-short multiple-nap protocol study (20/40 min sleep/wake schedules) (Suzuki et al., 2004). Dreaming scores from NREM sleep reports were sinusoidally distributed across the 24-h day, with maxima at 08:00 h. REM sleep report scores were high during the diurnal period of 06:00-16:00 $\mathrm{h}$ and then dropped. Of particular interest, the NREM sleep dream score peak coincided with the peak of REM sleep stage duration. One likely explanation could be that human dream experiences in NREM sleep are influenced by the REM sleep-generating mechanisms (Nielsen, 2004). Ponto-geniculo-occipital activity, a robust factor activating the visual cortices that generates dream experiences, and a hallmark of REM sleep (Hobson and Mc Carley, 1977), may occur in human NREM sleep, particularly in the morning hours when REM sleep is most likely to occur, thus resulting in dream experiences during NREM sleep (Suzuki et al., 2004). REM sleep propensity is under tight circadian control, and exhibits a clear peak in the early morning hours (Dijk et al., 1997). Under this assumption, one may hypothesize that the coincident NREM sleep dream peak with the peak of REM sleep stage duration described in Suzuki et al. (2004) suggests that the dreaming propensity from both REM and NREM sleep is influenced by the circadian oscillator.

In the next section, we address recent studies on how dreaming is modulated by the NREM-REM sleep regulation at an electrophysiological level, and what is the role of the circadian system on dream recall.

\section{The NREM-REM sleep and the circadian signatures of dreaming}

Recently we investigated the electrophysiological NREM and REM sleep EEG correlates prior to dream recall and age-related effects on dream recall (Chellappa et al., 2009, 2011, 2012). EEG power density during sleep associated with and without dream recall was investigated in both young and older participants. Furthermore, we focused on how the topographic distribution of EEG activity during NREM and REM sleep can underpin potential cortical networks related to dream recall. The methodological rationale was to access dream recall using the sleep mentation questionnaire (Chellappa et al., 2009), which was carried out immediately after awakenings from scheduled 75-min naps during a 40-h multiple nap paradigm (150/75 min of wake-sleep schedule; 10 naps in total), under constant routine conditions (Cajochen et al., 2006). This enabled the estimation of dream 
recall after multiple sleep episodes, without the experimental bias of awakening participants during, for instance, an 8-h sleep episode, which is the most usual approach in dream research. Furthermore, this circadian-driven procedure allowed quantifying the contribution of the circadian timing system on dream recall.

In young individuals, dream recall was associated with frequencyand topography specific spectral EEG activity during NREM and REM sleep. During NREM sleep, dream recall was linked to less frontal delta (1-3 Hz, Fig. 1, left panel) and centro-parietal spindle (12-15.5 Hz) activities (Chellappa et al., 2011). These effects might be explained by the intrinsic thalamocortical network during NREM sleep, as indexed by higher levels of sleep spindles and delta waves, which hamper ongoing mental activity (Steriade et al., 1993). At the transition from wakefulness to sleep, the neuronal membrane potential in the cortex and thalamus, the relay station for most sensory signals to the cerebral cortex, reduces, resulting in NREM sleep oscillations - sleep spindles and slow-waves - with impaired synaptic responsiveness (Timofeev et al., 2001). Thus, it is very likely that this neuronal network explains why dream recall was reduced during delta and spindle activity. NREM sleep frequency-specific differences also displayed clear biological day-night differences, such that dream recall was associated to lower delta and spindle activity, while no dream recall was related to higher spindle activity during the biological day (Chellappa et al., 2011). Evidence supports that the circadian modulation of spindle frequency is phase-locked with melatonin secretion, a classical marker of the circadian clock (Knoblauch et al., 2005), with, respectively, higher spindle activity during the biological night and lower spindle activity during the biological day. This type of circadian modulation of spindle frequency would then facilitate consolidated night sleep and day wakefulness (Knoblauch et al., 2005). In this context, daytime fast spindle frequencies can represent a circadian wake promoting signal, resulting thus in less dream recall following NREM sleep during the day.

In young individuals, dream recall following REM sleep was higher than after NREM sleep (Fig. 2, left panel). Furthermore, dream recall was associated with lower REM alpha activity (10-12 Hz, Fig. 1, right panel) (Chellappa et al., 2011). Dream recall has been associated with alpha activity in a non-topographic manner (Esposito et al., 2004), and argued as a means to cognitive elaboration active prior to awakening. Interestingly, we observed less alpha activity only in frontal derivations, which hints to a "prefrontal cortex deactivation" during REM dreaming (Maquet et al., 1996), resulting in less higher order analytic reasoning thinking. Similar as for NREM sleep, the REM sleep frequency-specific differences showed clear biological day-night differences, such that successful dream recall was associated to higher alpha activity during the biological day (Chellappa et al., 2011). Interestingly, we observed that the circadian modulation of dream recall was closely associated with the time course of REM sleep across the naps, with highest levels of dream recall in the morning (05:00-09:00 h) (Fig. 3) (Chellappa et al., 2009). The circadian modulation of alpha activity during REM sleep is such that it coincides with the end of the time window when melatonin levels are high (close to the crest of the REM sleep propensity, during the early morning hours (Dijk et al., 1997; De Gennaro and Ferrara, 2003)). This in turn may result in higher levels of dream recall associated to REM alpha activity, during the biological day.

While dreaming in young individuals has received most of the attention in dream research, relatively little is known about age-related differences in dreaming. This is our focus for the next section.

\section{An old question in dream research: Aging and dream recall}

Aging is associated with significantly less dream recall (Waterman, 1999; Zanasi et al., 2005). Since the circadian timing system seems to modulate dream recall, age-related changes within this system are a plausible candidate to explain changes in dream recall. Older people exhibit circadian rhythms of core body temperature and melatonin rhythms with attenuated amplitude (Münch et al., 2005) and/or phase advanced (Dijk et al., 1999; Yoon et al., 2003). Furthermore, an age-related decrease in the amplitude of the circadian signal results in a dampening of the circadian drive for wakefulness in the evening hours and for sleep in the morning hours (Münch et al., 2005). This may result in an internal circadian advance, relative to the rhythm of core body temperature and melatonin, of the propensity to awaken in older people (Duffy et al., 2002). As a consequence, retrospectively estimated dream recall in older healthy volunteers is reduced (Fig. 2, right panel) (Chellappa et al., 2009). In one of our previous studies (Chellappa et al., 2009), we observed that older individuals had an earlier peak of the emotional score of their dream recall, as compared to the young, during the morning (05:00 $\mathrm{h}$ in the older, compared to 09:00 $\mathrm{h}$ in the younger), suggesting that older individuals may exhibit
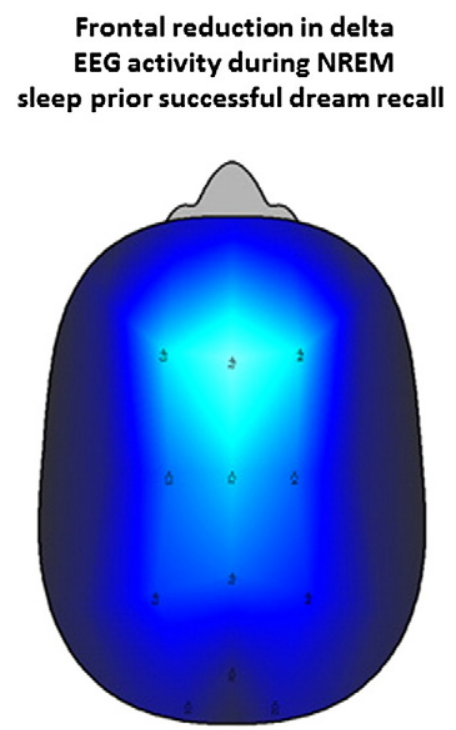
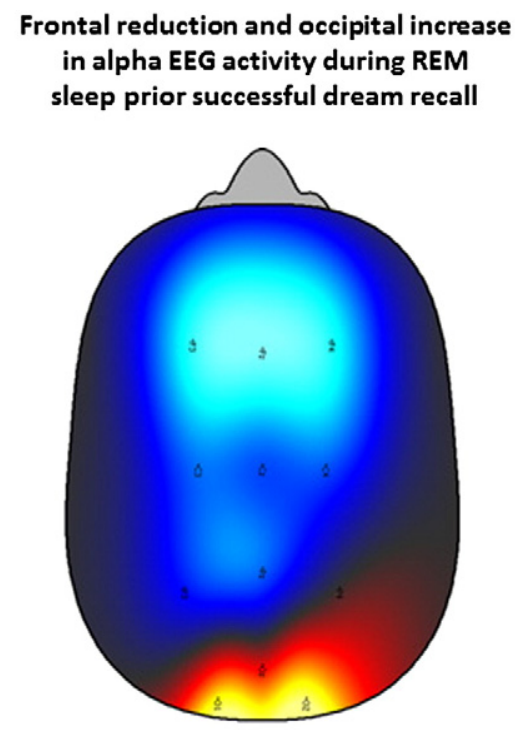

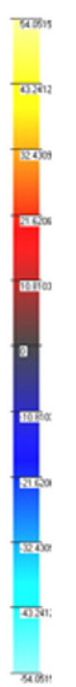

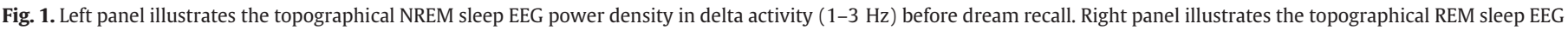

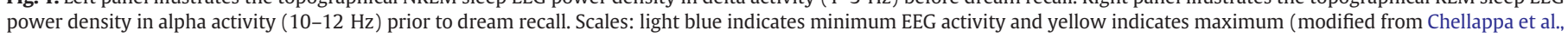
2011). 


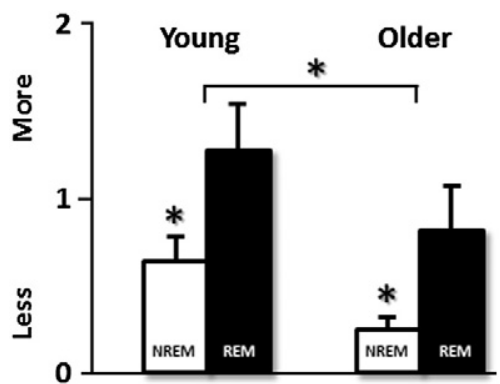

Fig. 2. Dream recall for young and older volunteers subsequent to NREM (white bars) and REM naps (black bars) and older volunteers (black bars). Scores are presented as mean values \pm SEM, ${ }^{*} p<0.05$ (modified from Chellappa et al., 2009).

a phase advance in their retrospectively estimated dream recall at least for the emotional domain. Another likely reason for this agerelated decline in dream recall could be the attenuation of REM sleep, which significantly decreases during middle-aged adulthood, and remains relatively stable in individuals older than 60 years (Ohayon et al., 2004).

With respect to the electrophysiological correlates of sleep before dream recall, aging was linked to specific frequency- and topography EEG activity prior to dream recall, such that during NREM sleep, older people had more frontal delta and centro-parietal sigma activity before dream recall (Chellappa et al., 2012). This paradoxical finding could be due to an increase in NREM sleep stage 2, which is the NREM sleep stage mostly connected to dream recall (Takeuchi et al., 2003). The absence of clear differences between REM sleep spectral composition before dream recall and no recall in older people may reflect a global attenuation of REM sleep (Münch et al., 2005; Chellappa et al., 2009). However, since aging was associated with less frontal alpha and beta activity (irrespective of dreaming or not) (Chellappa et al., 2012), it is tempting to speculate that REM dreaming in older people does not affect the emotional tone of dreaming, but rather the analytic thinking domain, due to a reduced activation of the frontal cortex (Braun et al., 1997).

Taken together, the critical insight from sleep data is that the key to unlock what dreaming means is to understand what the neuronal correlates of dreaming are.

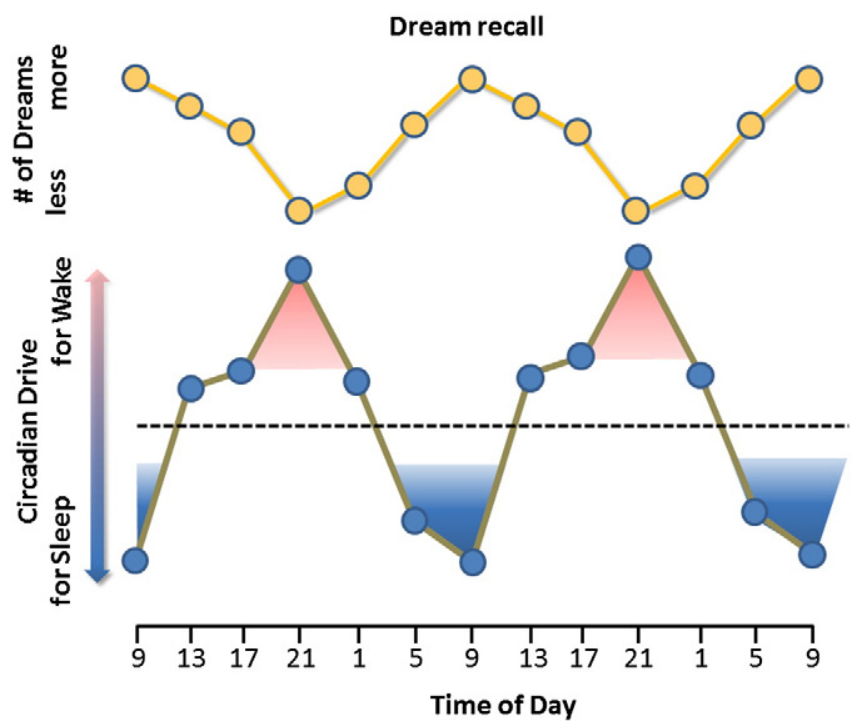

Fig. 3. Time course of dream recall for young volunteers (upper panel) and the dynamics of the circadian drive for sleep and wakefulness (lower panel) (modified from Chellappa et al., 2009).

\section{Neuroimaging insights on dreaming}

The last two decades have experienced an exponential interest in the field of neuroimaging and dreaming (for a review, see Kussé et al., 2010). One straightforward approach has been to investigate the underlying regional brain function during sleep with brain imaging techniques (Schwartz and Maquet, 2002). NREM sleep comprises a state of vigilance characterized by a set of oscillations that modulate neural firing in specific ways (slow oscillation, slow-waves, spindles), gated by specific regional brain responses and associated to specific functional processes (Kussé et al., 2010). Thus, if sleep results in a retrievable imagery representation, such as dreaming, the conclusion is that the distribution of regional brain function is intertwined to these ongoing imagery mental processes.

To the best of our knowledge, functional neuroimaging characterization of dream correlates has been conducted only during REM sleep (Kussé et al., 2010). REM sleep is linked to an increase in regional brain activity in the pontine tegmentum, thalamus, basal forebrain, as well as in limbic and paralimbic structures, including amygdaloid complexes, hippocampal formation, and anterior cingulate cortex (ACC) (Maquet et al., 1996; Nofzinger et al., 1997; Braun et al., 1997). Activation in these regions hints to the possibility that memory consolidation processes, particularly emotional memories, may happen during REM sleep (Wagner et al., 2001; Nishida et al., 2009; Sterpenich et al., 2009; Walker, 2009). Conversely, numerous key regions are hypoactive during REM sleep relative to wake state, such as the dorsolateral prefrontal cortex (DLPFC), orbitofrontal cortex, posterior cingulate gyrus, precuneus, and the inferior parietal cortex (Maquet et al., 1996; Nofzinger et al., 1997; Braun et al., 1997; Maquet, 2000). Deactivations in these regions that subserve critical executive and attention functions during wake state point to the fact that the functional neuroanatomy of REM sleep is diametrically apart from that of wake state (Maquet et al., 1996; Nofzinger et al., 1997; Braun et al., 1997; Maquet, 2000).

Conversely, NREM sleep is associated to widespread cerebral deactivation, including the pontine brainstem, orbito-frontal cortex and anterior cingular cortex (Braun et al., 1997; Hofle et al., 1997), while REM sleep is linked to the activation of these same regions (Maquet et al., 1996). This NREM sleep-driven deactivation thus reflects the continuous decline in the activation of the reticular activating system (RAS), and results in the disfacilitation of thalamocortical relay neurons, which then allows the emergence of thalamocortical oscillations (Steriade, 1997). NREM deactivation of ascending arousal systems, such as the pons and midbrain, may probably translate into overall lower levels of global forebrain activation (Hofle et al., 1997; Maquet et al., 1997) and a concomitant decrease in cognitive output from the cerebral cortex. Moreover, the deactivation of limbic structures, such as the anterior cingulate (Hofle et al., 1997; Hobson et al., 1998), may hinder the emotional salience as compared to REM sleep. In a broader context, REM and NREM sleep display specific regional changes in brain activity and functional neural connectivity that may pinpoint to the differences in dreaming.

\section{Summary and perspectives on dreaming}

We provided evidence in favor of a circadian and NREM/REM sleep modulation for dreaming, reflected by the association of dream recall to the circadian modulation of REM sleep, biological day-night differences, and the striking differences in NREM and REM sleep EEG activity before dream recall (Chellappa et al., 2009, 2011, 2012). Furthermore, we showed that circadian and NREM-REM sleep modulation do not remain constant throughout a life span, as indexed by the sleep electrophysiological changes before dream recall in older people (Chellappa et al., 2012). These specific NREM and REM sleep frequency and topography differences prior to dream recall, together with neuroimaging 
data, provide a scenario in which regional brain activation during sleep is directly implied in dreaming. A way forward to discover the mechanisms that underlie dreaming will be to better understand the specific cerebral correlates of quantitative (dream recall) and of qualitative (dream reports) aspects of dreaming, across different sleep states and also during quiet wakefulness ("daydreams"). Recent evidence indicates that sleep dreaming and quiet wakefulness daydreaming share similar features (emotional salience and projection of past events onto the future) (Wamsley et al., 2010). This suggests common underlying cortical mechanisms accounting for these mnemonic processes, such as the default-mode network (Wamsley et al., 2010). Therefore, dreams and daydreams may represent a continuum of subjective experience across different states of vigilance, and not two unrelated events. Ultimately, knowledge on dream generation, by means of multiple techniques like EEG and fMRI, may shed light on one of the oldest questions of humanity, "why do we dream?"

\section{References}

Antrobus, J., Kondo, T., Reinsel, R., 1995. Dreaming in the late morning: summation of REM and diurnal cortical activation. Consciousness and Cognition 4, 275-299.

Armitage, R., Hoffmann, R., Loewy, D., Moffitt, A., 1989. Variations in period-analysed EEG asymmetry in REM and NREM sleep. Psychophysiology 26, 329-336.

Aserinsky, E., Kleitman, N., 1953. Regularly occurring periods of eye motility, and concomitant phenomena, during sleep. Science 118, 273-274.

Braun, A.R., Balkin, T.J., Wesensten, N.J., Carson, R.E., Varga, M., Baldwin, P., 1997. Regional cerebral blood flow throughout the sleep-wake cycle. An $\mathrm{H}_{2}^{15} \mathrm{O}$ PET study. Brain 120, 1173-1197.

Cajochen, C., Knoblauch, V., Kräuchi, K., Schröder, C., Wirz-Justice, A., 2003. Circadian modulation and EEG correlates of dream recall during a 75:150-min sleep-wake cycle paradigm. Sleep 26, 93.

Cajochen, C., Münch, M., Knoblauch, V., Blatter, K., Wirz-Justice, A., 2006. Age-related changes in the circadian and homeostatic regulation of human sleep. Chronobiology International 23, 1-14.

Cartwright, R., Luten, A., Young, M., Mercer, P., Bears, M., 1997a. Role of REM sleep and dream affect in overnight mood regulation: a study of normal volunteers. Psychiatric Research 81, 1-8.

Cartwright, R., Young, M.A., Mercer, P., Bears, M., 1997b. Role of REM sleep and dream variables in the prediction of remission from depression. Psychiatric Research 80, 249-255.

Casagrande, M., Violani, C., Lucidi, F., Buttinelli, E., Bertini, M., 1996. Variations in sleep mentation as a function of time of night. International Journal of Neuroscience 85 , $19-30$.

Cavallero, C., Foulkes, D., Hollifield, M., Terry, R., 1990. Memory sources of REM and NREM dreams. Sleep 13, 449-455.

Chellappa, S.L., Münch, M., Blatter, K., Knoblauch, V., Cajochen, C., 2009. Does the circadian modulation of dream recall modify with age? Sleep 32, 1201-1209.

Chellappa, S.L., Frey, S., Knoblauch, V., Cajochen, C., 2011. Cortical activation patterns herald successful dream recall after NREM and REM sleep. Biological Psychology 87, 251-256.

Chellappa, S.L., Münch, M., Knoblauch, V., Cajochen, C., 2012. Age effects on spectral EEG activity prior to dream recall. Journal of Sleep Research 21, 247-256

Czaya, J., Kramer, M., Roth, T., 1973. Changes in dream quality as a function of time into REM. Sleep Research Online 2, 122-125.

De Gennaro, L., Ferrara, M., 2003. Sleep spindles: an overview. Sleep Medicine Reviews 7, 423-440.

Dement, W., Kleitman, N., 1957. The relation of eye movements during sleep to dream activity: an objective method for the study of dreaming. Journal of Experimental Physiology 53, 339-346

Desseilles, M., Dang-Vu, T.T., Sterpenich, V., Schwartz, S., 2011. Cognitive and emotional processes during dreaming: a neuroimaging view. Consciousness and Cognition 20 998-1008.

Dijk, D.J., Shanahan, T.L., Duffy, J.F., Ronda, J.M., Czeisler, C.A., 1997. Variation of electroencephalographic activity during non-rapid eye movement and rapid eye movement sleep with phase of circadian melatonin rhythm in humans. Journal of Physiology 505, 851-858.

Dijk, D.J., Duffy, J.F., Riel, E., Shanahan, T.L., Czeisler, C.A., 1999. Ageing and the circadian and homeostatic regulation of human sleep during forced desynchrony of rest, melatonin and temperature rhythms. Journal of Physiology 516, 611-627.

Duffy, J.F., Zeitzer, J.M., Rimmer, D.W., Klerman, E.B., Dijk, D.J., Czeisler, C.A., 2002 Peak of circadian melatonin rhythm occurs later within the sleep of older subjects. American Journal of Physiology, Endocrinology and Metabolism 282, 297-303.

Esposito, M.J., Nielsen, T.A., Paquette, T., 2004. Reduced Alpha power associated with the recall of mentation from stage 2 and stage REM sleep. Psychophysiology 41 , 288-297.

Foulkes, D., 1993. Normal and abnormal REM sleep regulation: dreaming and REM sleep. Journal of Sleep Research 2, 199-202.
Hobson, J.A., Mc Carley, R.W., 1977. The brain as a dream state generator: an activationsynthesis hypothesis of the dream process. American Journal of Psychiatry 134, 1335-1348.

Hobson, J.A., Pace-Schott, E.F., Stickgold, R., Kahn, D., 1998. To dream or not to dream? Relevant data from new neuroimaging and electrophysiological studies. Current Opinion in Neurobiology 8, 239-244.

Hobson, J.A., Pace-Schott, E.F., Stickgold, R., 2000. Dreaming and the brain: toward a cognitive neuroscience of conscious states. The Behavioral and Brain Sciences 23, 793-842.

Hofle, N., Paus, T., Reutens, D., Fiset, P., Gotman, J., Evans, A.C., 1997. Regional cerebral blood flow changes as a function of delta and spindle activity during slow wave sleep in humans. Journal of Neuroscience 17 (12), 4800-4808.

Knoblauch, V., Münch, M., Blatter, K., Wirz-Justice, A., Cajochen, C., 2005. Age-related changes in the circadian modulation of sleep-spindle frequency during nap sleep. Sleep 28, 1093-1101.

Kussé, C., Muto, V., Mascetti, L., Matarazzo, L., Foret, A., Shaffi-Le Bourdiec, A., Maquet, P., 2010. Neuroimaging of dreaming: state of the art and limitations. International Journal of Neurobiology 92, 87-99.

Lehmann, D., Dumermuth, G., Lange, B., Meier, C., 1981. Dream recall related to EEG spectral power during REM periods. Sleep Research 10, 191-192.

Llinás, R., Ribary, U., 1993. Coherent $40-\mathrm{Hz}$ oscillation characterizes dream state in humans. PNAS 90, 2078-2081.

Maquet, P., 2000. Functional neuroimaging of normal human sleep by positron emission tomography. Journal of Sleep Research 9, 207-231.

Maquet, P., Peters, J.M., Aerts, J., Delfiore, G., Degueldre, C., Luxen, A., 1996. Functional neuroanatomy of human rapid-eye movement sleep and dreaming. Nature 383, $163-164$.

Maquet, P., Degueldre, C., Delfiore, G., Aerts, J., Péters, J.M., Luxen, A., 1997. Functional neuroanatomy of human slow wave sleep. Journal of Neuroscience 17 , 2807-2812.

Marzano, C., Ferrara, M., Mauro, F., Moroni, F., Gorgoni, M., Tempesta, D., Cipolli, C., De Gennaro, L., 2011. Recalling and forgetting dreams: theta and alpha oscillations during sleep predict subsequent dream recall. Journal of Neuroscience 31, 6674-6683.

Münch, M., Knoblauch, V., Blatter, K., Schröder, C., Schnitzler-Sack, C., Kräuchi, K., WirzJustice, A., Cajochen, C., 2005. Age-related attenuation of the evening circadian arousal signal in humans. Neurobiology of Aging 26, 1307-1319.

Nielsen, T.A., 2000. A review of mentation in REM and NREM sleep: “covert" REM sleep as a possible reconciliation of two opposing models. The Behavioral and Brain Sciences $23,851-866$

Nielsen, T.A., 2004. Chronobiological features of dream production. Sleep Medicine Reviews 8, 403-424.

Nielsen, T.A., Chénier, V. 1999. Variations in EEG coherence as an index of the affective content of dreams from REM sleep: relationships with face imagery. Brain and Cognition 41, 200-212.

Nishida, M., Pearsall, J., Buckner, R.L., Walker, M.P., 2009. REM sleep, prefrontal theta, and the consolidation of human emotional memory. Cerebral Cortex 19, 1158-1166.

Nofzinger, E.A., Mintun, M.A., Wiseman, M., Kupfer, D.J., Moore, R.Y., 1997. Forebrain activation in REM sleep: an FDG PET study. Brain Research 770, 192-201.

Ohayon, M., Carskadon, M., Guilleminault, C., Vitiello, M., 2004. Meta-analysis of quantitative sleep parameters from childhood to old age in healthy individuals: developing normative sleep values across the human lifespan. Sleep 27, 1255-1265.

Schwartz, S., Maquet, P., 2002. Sleep imaging and the neuropsychological assessment of dreams. Trends in Cognitive Sciences 6, 23-30.

Steriade, M., 1997. Synchronized activities of coupled oscillators in the cerebral cortex and thalamus at different levels of vigilance. Cerebral Cortex 7 . 583-604.

Steriade, M., McCormick, D.A., Sejnowski, T.J., 1993. Thalamocortical oscillations in the sleeping and aroused brain. Science 262, 679-685.

Sterpenich, V., Albouy, G., Darsaud, A., Schmidt, C., Vandewalle, G., Dang Vu, T.T., Luxen, A., Maquet, P., 2009. Sleep promotes the neural reorganization of remote emotional memory. Journal of Neuroscience 29, 5143-5152.

Stickgold, R., Malia, A., Fosse, R., Propper, R., Hobson, J.A., 2001a. Brain-mind states: I. longitudinal field study of sleep/wake factors influencing mentation report length. Sleep 24, 71-79.

Stickgold, R., Hobson, J., Fosse, R., Fosse, M., 2001b. Sleep, learning, and dreams: off-line memory reprocessing. Science $294,1052-1057$.

Suzuki, H., Uchiyama, M., Tagaya, H., Ozaki, A., Kuriyama, K., Aritake, S., 2004. Dreaming during non-rapid eye movement sleep in the absence of prior rapid eye movement sleep. Sleep 27, 1486-1490.

Takeuchi, T., Ogilviec, R.D., Murphy, T.I., Ferrelli, A.V., 2003. EEG activities during elicited sleep onset REM and NREM periods reflect different mechanisms of dream generation. Clinical Neurophysiology 114, 210-220.

Timofeev, I., Grenier, F., Steriade, M., 2001. Disfacilitation and active inhibition in the neocortex during the natural sleep-wake cycle: an intracellular. PNAS 98, 1924-1929.

Wagner, U., Gais, S., Born, J., 2001. Emotional memory formation is enhanced across sleep intervals with high amounts of rapid eye movement sleep. Learning Memory 8, 112-119.

Walker, M.P. 2009. The role of sleep in cognition and emotion. Annals of the New York Academy of Sciences 1156, 168-197.

Wamsley, E.J., Hirota, Y., Tucker, M.A., Smith, M.R., Antrobus, J.S., 2007. Circadian and ultradian influences on dreaming: a dual rhythm model. Brain Research Bulletin $71,347-354$ 
Wamsley, E.J., Tucker, M., Payne, J.D., Benavides, J.A., Stickgold, R., 2010. Dreaming of a learning task is associated with enhanced sleep-dependent memory consolidation. Current Biology 20, 850-855.

Waterman, D., 1999. Aging and memory for dreams. Perceptual and Motor Skills 73, 355-365.

Williamson, P.C., Csima, A., Galin, H., Mamelak, M., 1986. Spectral EEG correlates of dream recall. Biological Psychiatry 21, 717-723.
Yoon, I.Y., Kripke, D.F., Elliott, J.A., Youngstedt, S.D., Rex, K.M., Hauger, R.L., 2003. Agerelated changes of circadian rhythms and sleep-wake cycles. Journal of the American Geriatrics Society 51, 1085-1091.

Zanasi, M., De Persis, S., Caporali, M., Siracusano, A., 2005. Dreams and age. Perceptual and Motor Skills 100, 925-938. 